\title{
Addition of trazodone to sertraline: a probable synergistic action in a case of obsessive- compulsive disorder
}

Adição de trazodona a sertralina: uma provável ação sinérgica em um caso de transtorno obsessivo-compulsivo

\section{Dear Editor,}

Despite the considerable advances made with the introduction of the serotonin reuptake inhibitors (SRIs) into clinical practice, $40-60 \%$ of the subjects still fail to respond adequately to the initial therapy. Preliminary evidence supports the addition of antipsychotics, mainly atypical, to SRIs in obsessive-compulsive disorder (OCD). These agents combine serotonin-dopamine antagonism with the advantage of being well tolerated, including a low potential for inducing motor side-effects. In contrast, other serotonergic medications do not show satisfactory results as adjuvant treatments in treatment-refractory OCD. ${ }^{1}$

\section{Case report}

A 37 year-old white woman was admitted to our department with a ten-year history of obsessive-compulsive symptoms 
characterized by mild repetitive religious thoughts, as obsessions, and extreme cleaning and repeating behaviors, as compulsions. When she took a shower she started by washing each side of her right leg exactly 45 times, followed by her left leg. She consistently combed her hair exactly 45 times on each side and washed her hands unnecessarily more than 20 times a day causing a severe dermatitis due to this excessive washing. She was aware that his compulsions were excessive and caused marked distress in her life. She scored 25 (7 for obsessions and 18 for compulsions) on the Yale-Brown Obsessive Compulsive Scale (YBOCS).

Of note in her past medical history, she has a twenty-two-year history of myasthenia gravis that has been treated with prednisolone $(80 \mathrm{mg} /$ day) with stability of symptoms such as fatigable weakness and fluctuating diplopia.

The patient was adequately treated with sertraline (200 mg/day) during six months with decrease of the YBOCS's score to 11 (3 for obsessions and 8 for compulsions), which was associated with great improvement in her quality of life.

During the ninth month of sertraline's use, she started to complain about initial insomnia. Sleep hygiene did not demonstrate improvement. Due to myasthenia gravis, trazodone $100 \mathrm{mg}$ at night was introduced, with good response. However, after three weeks, at her clinical evaluation, her OCD symptoms showed further remission, and she scored 4 on the YBOCS (one point for obsessions and three for compulsions).

\section{Discussion}

Trazodone is an atypical antidepressant drug whose most potent pharmacological effect appears to be an antagonist action at 5HT2/1C receptors ${ }^{2}$ in contrast to sertraline, for which inhibition of 5 -HT uptake is the most potent pharmacological action. ${ }^{1}$

In the literature, when trazodone is managed as the only medication for the treatment of OCD, a study evidenced significant reduction in the obsessive-compulsive symptoms in $75 \%$ of the patients, ${ }^{3}$ while two others, ${ }^{4,5}$ one of them a double-blind, placebo controlled study, ${ }^{4}$ indicated that trazodone lacks substantial antiobsessive compulsive effects. However, some case reports have demonstrated a rapid and impressive improvement in OCD symptoms after introducing trazodone while using a SRI, probably due to a synergistic action of both (via inhibition of 5-HT uptake and antagonist action at 5-HT2/1C receptors). ${ }^{1,2}$

Despite preliminary, the association of a SRI, in our case, sertraline, with trazodone seems to be a promising strategy in the treatment of OCD. Furthermore, its use in depression seems to be well tolerated and safe. ${ }^{1}$ However, controlled studies are necessary to better define its role in this clinical challenging condition.

Felipe Filardi da Rocha, Humberto Corrêa Department of Psychiatry, School of Medicine, Universidade Federal de Minas Gerais (UFMG), Belo Horizonte (MG), Brazil

Naira Vassalo Lage Department of Occupational Therapy, School of Medicine, Universidade Federal de Minas Gerais (UFMG), Belo Horizonte (MG), Brazil

\section{References}

1. Blier P, Habib R, Flament MF. Pharmacotherapies in the management of obsessive-compulsive disorder. Can J Psychiatry. 2006;51(7):417-30

2. Hermesh $H$, Aizenberg D, Munitz $H$. Trazodone treatment in clomipramine-resistant obsessive-compulsive disorder. Clin Neuropharmacol. 1990;13(4):322-8.

3. Pigott TA, L'Heureux F, Rubenstein CS, Bernstein SE, Hill JL, Murphy $\mathrm{DL}$. A double-blind, placebo controlled study of trazodone in patients with obsessive-compulsive disorder. J Clin Psychopharmacol. 1992;12(3): 156-62.

4. Prasad A. Efficacy of trazodone as an anti-obsessional agent. Neuropsychobiology. 1986;15 Suppl 1:19-21.

5. Sunkureddi K, Markovitz P. Trazodone treatment of obsessivecompulsive disorder and trichotillomania. Am J Psychiatry. 1993;150(3):523-4. 